\title{
Physical parameters for RR Lyrae stars in the SDSS filter system
}

\author{
Márcio Catelan, ${ }^{1,2}$ Gabriel I. Torrealba, ${ }^{1,2}$ Claudio Cáceres, ${ }^{1,2,3}$ \\ Horace A. Smith, ${ }^{4}$ Nathan De Lee, ${ }^{5}$ and Michael Fitzgerald ${ }^{6}$ \\ ${ }^{1}$ Departamento de Astronomía y Astrofísica, Pontificia Universidad Católica de Chile, \\ Av. Vicuña Mackenna 4860, 782-0436 Macul, Santiago, Chile \\ email: mcatelan@astro.puc.cl \\ ${ }^{2}$ The Milky Way Millennium Nucleus, Santiago, Chile \\ ${ }^{3}$ Departamento de Física y Astronomía, Universidad de Valparaíso, Av. Gran Bretaña 1111, \\ Playa Ancha, Valparaíso, Chile \\ ${ }^{4}$ Department of Physics and Astronomy, Michigan State University, East Lansing, MI 48824, \\ USA \\ ${ }^{5}$ Department of Physics and Astronomy, Vanderbilt University, Nashville, TN 37235, USA \\ ${ }^{6}$ Department of Physics \& Astronomy, Macquarie University, NSW 2109, Australia
}

\begin{abstract}
We present a calibration of the metallicity and physical parameters (temperature, luminosity, gravity, mass, radius) for RR Lyrae stars using the ugriz SDSS photometric system. Our work is based on calculations of synthetic horizontal branches (HBs), fully taking into account evolutionary effects for a wide range in metallicities and HB morphologies. We provide analytical fits that are able to provide all quantities mentioned with very high (internal) precision, based solely on mean SDSS magnitudes and colors.
\end{abstract}

Keywords. stars: fundamental parameters, stars: horizontal-branch, stars: variables: other

\section{Introduction}

The Sloan Digital Sky Survey (SDSs; York et al. 2000; Aihara et al. 2011) represents the dawn of a new era in astronomy, in which wide-field sky surveys play increasingly important roles. Such surveys are of key importance for our understanding of structure in the Universe, both locally and at cosmological distances. In this sense, it is particularly important that the properties of key distance indicators, such as RR Lyrae stars, are well-understood and properly calibrated on the basis of SDSS photometric indices.

In the wake of the SDSS survey itself, the SDSS photometric system (Fukugita et al. 1996, 2011) has gained much visibility, and sDSs filters are now commonly available at all major observatories. Indeed, many of the current and future wide-field dedicated telescopes and surveys, including the Large Synoptic Survey Telescope (LSST; Ivezić et al. 2008b), PanSTARRS (Kaiser et al. 2002; Stubbs et al. 2007), the VLT Survey Telescope (VST; Kuijken et al. 2002), the Dark Energy Survey (DES; Tucker et al. 2007), and SkyMapper (Keller et al. 2007; Bessell et al. 2011), will be (or are already) carried out using filter systems that generally bear close resemblance to the original SDSs system.

While it is known that the SDSS system possesses great scientific potential for a variety of science applications, including stellar populations (e.g., Lenz et al. 1998; Helmi et al. 2003; Ivezić et al. 2008a; Lardo et al. 2011; Vickers et al. 2012), the behavior of variable stars in general in such filter systems has not yet been as extensively studied as for more traditional filter systems, particularly the Johnson-Cousins system. For instance, no systematic studies of RR Lyrae variability in globular clusters has yet been carried out in 
the SDSS system, and similarly theoretical analyses of RR Lyrae variable stars in the SDSS system are almost entirely lacking, with the studies of Marconi et al. (2006) and Cáceres \& Catelan (2008) seemingly representing the sole exceptions. To extract the maximum amount of information from extensive RR Lyrae databases that are increasingly becoming available in the SDSS (or similar) systems (e.g., Sesar 2011; Sesar et al. 2007, 2010, 2011), more extensive theoretical analyses are clearly needed.

In this sense, we recently started a systematic study, based on theoretical models and synthetic calculations for horizontal-branch (HB) stars, to define precise relations that should allow one to calculate distances, reddening and metallicity values, and physical parameters of RR Lyrae stars from SDss photometric observations. In Cáceres \& Catelan (2008) we presented the first detailed calibration of the RR Lyrae period-luminosity (PL) and period-color (PC) relations in the sDss system.

One shortcoming of the Cáceres \& Catelan (2008) PL and PC calibrations is that they require a priori knowledge of the metallicity, which is frequently not available, especially for field stars. Currently this requires either spectroscopic information or Fourier decomposition of $V$-band light curves, whose parameters have been calibrated in terms of metallicity (Jurcsik \& Kovács 1996; Morgan et al. 2007). However, as pointed out by Jurcsik \& Kovács (1996), such calibrations of Fourier-decomposition parameters are not applicable to all RR Lyrae stars, requiring very well-behaved light curves. In addition, exceedingly complete phase coverage is required for the computation of reliable Fourier parameters. Therefore, calibrations that are based solely on the average photometric properties of the RR Lyrae stars are certainly desired.

The main purpose of the present project is thus to extend the Cáceres \& Catelan (2008) study, by providing an additional set of analytical expressions that allow one to compute metallicities, luminosities, temperatures, masses, gravities, and radii of RR Lyrae stars, solely on the basis of their average photometric properties. Our numerical experiments have shown that it is possible to derive fairly precise relations in the multiband SDSS and Strömgren (1963) systems, whereas we have not achieved similar success for the Johnson-Cousins $U B V R I$ system.

\section{Models}

The HB simulations computed in the present paper follow the same techniques as described in Catelan (2004) and Catelan et al. (2004), to which the reader is referred for further details and references about the HB synthesis method. In the present paper, we use the same HB simulations as already employed in our study of the PL and PC relations in the sDss filter system (Cáceres \& Catelan 2008).

\section{Pseudocolors for the SDSS System}

In this study, we define two pseudocolors in the SDSS system,

$$
\begin{aligned}
C_{0} & =(u-g)_{0}-(g-r)_{0} \\
m_{0} & =(g-r)_{0}-(r-i)_{0} .
\end{aligned}
$$

These indices are patterned after the well-known gravity and metallicity indices of the Strömgren (1963) system. Similarly to what happens in the latter system, both indices are fairly insensitive to reddening, with $E\left(C_{1}\right) \approx-0.32 E(B-V)$ and $E\left(m_{1}\right) \approx-0.38$ $E(B-V)$. Cáceres \& Catelan (2008) already showed that the introduction of $C_{0}$ allows one to compute precise relationships for the derivations of absolute magnitudes and colors 
in the SDSS system; we have found that $m_{0}$ is also very helpful, as far as derivation of metallicity and the remaining physical parameters of RR Lyrae stars is concerned.

\section{Results}

Full details of this investigation will be provided elsewhere; here we give only a brief preview of our results, showing a preliminary, small subsample of the relations derived so far. In all relations that follow, the (fundamentalized) period is given in days.

\subsection{Temperature}

$$
\log T_{\text {eff }}=\mathcal{A}+\mathcal{B}(g-r)_{0}+\mathcal{C} m_{0}+\mathcal{D} m_{0}^{2}+\mathcal{E}(g-r)_{0}(\log P)^{2},
$$

where $\mathcal{A}=3.84493, \mathcal{B}=-0.43149, \mathcal{C}=0.24311, \mathcal{D}=0.21078$, and $\mathcal{E}=0.15544$. The correlation coefficient is $\mathcal{R}=0.99997$, and the standard error of the estimate amounts to $\sigma=1.7 \times 10^{-4}$ dex.

\subsection{Gravity}

$$
\log g=\mathcal{A}+\mathcal{B} \log P+\mathcal{C} m_{0}+\mathcal{D}(g-r)_{0},
$$

where $\mathcal{A}=2.49342, \mathcal{B}=-1.22603, \mathcal{C}=-0.36479$, and $\mathcal{D}=0.24119$. The correlation coefficient is $\mathcal{R}=0.99995$, and the standard error of the estimate is $\sigma=0.0011$ dex.

\subsection{Luminosity}

$$
\begin{aligned}
\log \left(\frac{L}{\mathrm{~L}_{\odot}}\right)= & \mathcal{A}+\mathcal{B} M_{g}^{2} \log P+\mathcal{C} M_{g}+\mathcal{D}(g-r)_{0} \\
& +\mathcal{E} \log P+\mathcal{F} \log P(g-r)_{0}+\mathcal{G} m_{0}^{2},
\end{aligned}
$$

where $\mathcal{A}=1.78262, \mathcal{B}=0.07619, \mathcal{C}=-0.49604, \mathcal{D}=1.05627, \mathcal{E}=-0.40866, \mathcal{F}=$ $0.37410, \mathcal{G}=-1.21339$, and $M_{g}$ can be obtained from the equations provided in Cáceres \& Catelan (2008). The correlation coefficient here is $\mathcal{R}=0.99945$, and the standard error of the estimate is $\sigma=0.0019$ dex.

\subsection{Mass and Radius}

Note that, by using the previous equations, the definition of surface gravity, and the Stefan-Boltzmann law, one can also derive high-precision masses and radii. More specifically, masses derived on the basis of these expressions have an internal $\sigma=0.0017 \mathrm{M}_{\odot}$, whereas in the case of radii one has $\sigma=0.0026 \mathrm{R}_{\odot}$.

\subsection{Metallicity}

$$
[\mathrm{Fe} / \mathrm{H}]=\mathcal{A}+\mathcal{B} \frac{[\mathrm{Fe} / \mathrm{H}]_{0}^{2}}{(u-g)_{0}}+\mathcal{C} m_{0}(\log P)^{2}+\mathcal{D} m_{0}[\mathrm{Fe} / \mathrm{H}]_{0}^{2},
$$

with

$$
[\mathrm{Fe} / \mathrm{H}]_{0}=\mathcal{E}+\mathcal{F} C_{0}^{2} m_{0},
$$

where $\mathcal{A}=-0.78752, \mathcal{B}=-0.52606, \mathcal{C}=20.20356, \mathcal{D}=0.61526, \mathcal{E}=-3.29763$, and $\mathcal{F}=13.98842$. The correlation coefficient is $\mathcal{R}=0.99445$, and the standard error of the estimate is $\sigma=0.039$ dex. 


\subsection{Robustness of the Relations}

We have run extensive tests to check the robustness of the relations provided, based on independent sets of $\mathrm{HB}$ models covering a wider range of metallicities than originally used in Cáceres \& Catelan (2008), as well as empirical data, particularly for [Fe/H] (e.g., De Lee et al. 2007; De Lee 2008; and references therein). We find that the relations can be safely used, even outside their original range of applicability, provided that errors in the measurements of the colors and magnitudes of the 'equivalent static star' are not larger than 0.01-0.05 mag. In this context, work along the lines of Bono et al. (1995), but specifically focused on the SDSs filter system, is strongly encouraged.

\section{Acknowledgements}

This work is supported by the Chilean Ministry for the Economy, Development, and Tourism's Programa Iniciativa Científica Milenio (grant P07-021-F, awarded to The Milky Way Millennium Nucleus), by Proyecto Fondecyt Regular \#1110326, by the Center for Astrophysics and Associated Technologies (PFB-06), and by Proyecto Anillo ACT86. CC acknowledges support from ALMA-CONICYT project 31100025. HAS and NDL thank the US National Science Foundation for support under grant AST0707756.

\section{References}

Aihara, H., Allende Prieto, C., An, D., et al. 2011, ApJS, 193, 29; erratum: ApJS, 195, 26

Bessell, M., Bloxham, G., Schmidt, B., et al. 2011, PASP, 123, 789

Bono, G., Caputo, F., \& Stellingwerf, R. F. 1995, ApJS, 99, 263

Cáceres, C. \& Catelan, M. 2008, ApJS, 179, 242

Catelan, M. 2004, ApJ, 600, 409

Catelan, M., Pritzl, B. J., \& Smith, H. A. 2004, ApJS, 154, 633

De Lee, N. 2008, Ph.D. Thesis, Michigan State University, USA

De Lee, N. M., Smith, H. A., \& Beers, T. C. 2007, Bull. Am. Astron. Soc., 39, 776

Fukugita, M., Ichikawa, T., Gunn, J. E., et al. 1996, AJ, 111, 1748

Fukugita, M., Yasuda, N., Doi, M., Gunn, J. E., \& York, D. G. 2011, AJ, 141, 47

Helmi, A., Ivezić, Ž., Prada, F., et al. 2003, ApJ, 586, 195

Ivezić, Ž., Sesar, B., Jurić, M., et al. 2008a, ApJ, 684, 287

Ivezić, Ž, Tyson, J. A., Acosta, E., et al. 2008b, LSST: from Science Drivers to Reference Design and Anticipated Data Products (arXiv:0805.2366)

Jurcsik, J. \& Kovács, G. 1996, A\& A, 312, 111

Kaiser, N., Aussel, H., Burke, B. E., et al. 2002, Proc. SPIE, 4836, 154

Keller, S. C., Schmidt, B. P., Bessell, M. S., et al. 2007, Publ. Astron. Soc. Aus., 24, 1

Kuijken, K., Bender, R., Cappellaro, E., et al. 2002, The Messenger, 110, 15

Lardo, C., Bellazzini, M., Pancino, E., Carretta, E., Bragaglia, A., \& Dalessandro, E. 2011, $A \mathscr{E} A, 525, \mathrm{~A} 114$

Lenz, D. D., Newberg, J., Rosner, R., Richards, G. T., \& Stoughton, C. 1998, ApJS, 119, 121

Marconi, M., Cignoni, M., Di Criscienzo, M., et al. 2006, MNRAS, 371, 1503

Morgan, S. M., Wahl, J. N., \& Wieckhorst, R. M. 2007, MNRAS, 374, 1421

Sesar, B. 2011, Carnegie Obs. Astrophy. Ser., 5, 135

Sesar, B., Ivezić, Ž., Grammer, S. H., et al. 2010, ApJ, 708, 717

Sesar, B., Ivezić, Ž., Lupton, R. H., et al. 2007, AJ, 134, 2236

Sesar, B., Stuart, J. S., Ivezić, Ž., et al. 2011, AJ, 142, 190

Strömgren, B. 1963, QJRAS, 4, 8

Stubbs, C. W., High, F. W., George, M. R., et al. 2007, PASP, 119, 1163

Tucker, D. L., Annis, J. T., Lin, H., et al. 2007, in: The Future of Photometric, Spectrophotometric and Polarimetric Standardization (Sterken, C., ed.), ASP Conf. Ser., 364, 187

Vickers, J. J., Grebel, E. K., \& Huxor, A. P. 2012, AJ, 143, 86

York, D. G., Adelman, J., Anderson, J. E., Jr., et al. 2000, AJ, 120, 1579 